\title{
Does the Controllability of the Body Schema Predict Equilibrium in Elderly People? Characteristics of Relationships from a Lifelong Development Perspective
}

\section{Masayuki Watanabe*}

Department of Psychology, Faculty of Education, Shiga University, 2-5-1, Hiratsu, Otsu, 520-0862 Japan

*Corresponding author: Masayuki Watanabe, Department of Psychology, Faculty of Education, Shiga University, 2-5-1, Hiratsu, Otsu, 520-0862 Japan, Telephone: +81-77-537-7831; Fax : +81-77-537-7831; E-mail: watanabe@edu.shiga-u.ac.jp

Received date: July 21, 2018; Accepted date: August 27, 2018; Published date: August 31, 2018

Copyright: @2018 Watanabe M. This is an open-access article distributed under the terms of the Creative Commons Attribution License, which permits unrestricted use, distribution, and reproduction in any medium, provided the original author and source are credited.

\section{Abstract}

Background: Equilibrium is a primary factor that describes and predicts elderly people's falling and fear of falling. However, elucidation of the relationship between equilibrium and cognitive function and the influence of cognition function on equilibrium in elderly people is required.

Methods: This study sought to illustrate the characteristics of the equilibrium function in elderly people, focusing on the vividness and controllability of the body schema as a cognitive function and analyzing its relationship with body sway (in the one-leg standing test with eyes open) from a lifelong development perspective. Movement distance and the outer area in body sway in the one-leg standing test with eyes open were measured for 139 participants including children, college students, and elderly people. In addition, the vividness and controllability of the body schema were measured using reaction times in a video game-style imaginary body movement test.

Results: In the college students, both types of cognitive function were significantly correlated with equilibrium, indicating an association between these two variables. Moreover, different relationships between equilibrium and cognitive function were observed in children and elderly people. Therefore, equilibrium in the elderly people did not deteriorate to the extent that it reflected the characteristics of young children; rather, it was influenced by multiple factors that attributed to the deterioration of the motor organ while maintaining basic strategies continued from early adulthood.

Conclusion: Specific outcomes could be achieved to maintain the health of elderly people via future examining of the relationship between equilibrium and cognitive function.

Keywords: Body schema; Controllability; Equilibrium; Postural balance; Standing position; Vividness

\section{Introduction}

The deterioration of elderly people's performance and fitness increases their risk of falling and fracture, reduces their ability to perform activities of daily living, and diminishes their quality of life [1]. The annual fall rate for elderly people aged 65 years or older has reached $\geq 30 \%$ [2]. In addition, some elderly people experience postfall syndrome, which prevents walking despite the ability to walk without assistance [3]. Moreover, even if falls do not cause fractures, elderly people avoid leaving their homes [4]. The risk of falling related to reduced equilibrium is 2.9 times higher relative that related to other factors. Moreover, this risk increases to 5.0 times that for other risk factors in repeated falling [5]. In addition, equilibrium has been shown to be a stronger predictor of repeated falling relative to sex, age, fall frequency, mental function, and muscular strength [2]. Furthermore, decreased equilibrium is associated with post-fall syndrome [6]. Consequently, it is a primary factor in describing and predicting falls and fear of falling [7].

Reductions in equilibrium have been attributed to problems with the motor organs and are considered to originate from the brain functions responsible for coordination and decisions regarding physical behavior [8]. The relationship between equilibrium and cognitive function has recently received increased attention from this perspective. For example, Tabara et al. used the one-leg standing test with eyes open, which measures equilibrium, and showed that participants with standing times of $\leq 20 \mathrm{~s}$ were at greater risk of cerebral small vessel diseases and reduced cognitive function relative to those with longer standing times [9]. Moreover, Fujita et al reported that participants with greater working memory capacity exhibited stability in a one-leg standing test, even while performing cognitive tasks simultaneously [10].

However, Demnitz et al., who conducted a meta-analysis of the cross-sectional relationship between mobility and cognitive ability in elderly people, confirmed the conventional view that individuals with greater mobility exhibit higher levels of general cognition, executive function, memory, and processing, relative to those observed individuals with poor mobility, and there was no relationship between equilibrium and cognitive functions [11]. Therefore, findings regarding the relationship between equilibrium and cognitive function are inconsistent. Research is required to determine whether a relationship exists between these two functions on the condition that the specific content of each function is determined. 
In the equilibrium function, three types of sensory informationvisual, vestibular, and proprioceptive-are integrated by the central nerve in the brainstem and retained appropriately by controlling the body schema [12]. The body schema refers to a system of preconscious, sub personal processes that play a dynamic role in governing posture and movement [13]. The center of gravity can be maintained at the base of support through continuous minor adjustment to posture and physical movement, based on information relevant to the body schema [14]. This arousal and the operation of the body schema constitute a type of cognitive function. This function and equilibrium decline with age [15], which implies that there is a relationship between equilibrium and the body schema in elderly people.

It is necessary to measure the standards for each function accurately to verify the existence of this relationship. Equilibrium is generally measured using standing retention time in the standing test, measured via instruments such as the Mann Test and Berg Balance Scale. As one of various standing tests, the one-leg standing test (also referred to as the unipedal stance test or single-limb stance test) is a standardized field sobriety test to determine whether a DUI suspect is under the influence of alcohol or drugs. It is also a popular test for measuring balance, particularly as a simple assessment tool for balance in elderly people, because it can be used in a variety of settings and requires minimal equipment and training $[16,17]$.

It is also used to diagnose musculoskeletal ambulation disability symptom complex, which is a condition defined by three professional Japanese medical societies and involves symptoms of motor organ deterioration [18]. In addition, body sway in standing occurs in any direction within a range, but this type of complicated movement contains information that facilitates the analysis of related factors [19]. Fuchioka et al. showed that the center of pressure exerted a significant effect on gait speed in older adults. Therefore, the movement of the center of gravity became an index of quantitative information regarding equilibrium over a short period [20].

In addition, no standard method has been developed to measure the favorable body schema. Therefore, research should endeavor to understand the body schema from the perspectives of vividness and controllability with reference to the evaluation of motor images, similar to the body schema [21,22]. Vividness in motor imagery refers to the ability to generate vivid images or sensations of body images mentally, and controllability in motor imagery refers to the ability to manipulate the mental representation of body images. The one-leg standing test depends on static balance, which involves the retention of the center of physical pressure at the base of support, and dynamic balance, which involves returning the center of physical pressure to the base of support when it has moved [23].

As the vividness and controllability of the body schema respond to these postural control needs, it is appropriate to consider them an index of favorable body schema [24]. However, the body schema operates only under self-referential intentionality and does not present itself (vividness) intentionally, unlike motor images. In addition, the operation of motor images (controllability) does not become conscious. Therefore, the vividness of the body schema is defined as the ability to generate clear sensations of body schemas mentally, and controllability is the ability to manipulate sensations of body schemas mentally.

According to this definition, vividness and controllability in the body schema are equivalent to a cognitive function known as imaginary body movement, which refers to mental movement of an imaginary self (body schema) and becomes the core of the ability to obtain the spatial perspectives examined by Piaget and Inhelder using the "three mountains task" $[25,26]$. Conventionally, this was often measured via a true-or-false response to a statement regarding the way in which an object is seen from another perspective. However, with imaginary body movement, unique cognitive information processing, such as that involved in the distortion of the image of an object and the conversion of their positions, is included in the test. Watanabe created a method for the separate measurement of imaginary body movement, based on the fact that there is a linear function between the mental operation in imaginary body movement and response time.

The imaginary body movement test involves two conditions in which participants are seated on a chair with a back support (stable condition) or standing in an unstable position (unstable condition). Further, the function of arousal of the body schema (vividness) is used mainly in the stable condition, and the function of the operation of the body schema (controllability) is used mainly in the unstable condition. Therefore, the vividness and controllability of the body schema can be measured via the imaginary body movement test with stable and unstable conditions, in accordance with Watanabe [27].

Concerning the development of the vividness of the body schema, Schott measured motor images in elderly men in three age groups (i.e., $60-69,70-79$, and $\geq 80$ years) and young men younger than 20 years of age and reported that vividness did not differ significantly between the young men and those aged between 60 and 69 years but that observed in men aged 70 years or older was inferior to that observed in the young men [28]. Saimpont et al. also used paper questionnaires to measure the controllability of motor sense and visual images in young adults and elderly people and posited that controllability in elderly people was slightly inferior to that observed in the young adults [29]. Moreover, Watanabe and Takamatsu administered the imaginary body movement test to 428 participants ranging from children to elderly people [30].

In early childhood, vividness and controllability were largely inferior, relative to that observed in younger adults, but this difference decreased as the age of the adults increased. As described above, the vividness and controllability of the body schema increased rapidly from early childhood and gradually declined in late adulthood. However, Fujita et al. examined equilibrium by measuring body sway in adults aged between 22 and 88 years standing with their eyes closed and open. The results showed that while length per second increased with age and was predominantly positively correlated with age, length per area decreased as the participants' age increased [19]. In addition, the lengths per second and swinging area have been shown to decrease in a linear manner in children aged between 6 and 10 years standing on both legs [31]. Studies examining age-related changes in equilibrium in elderly people often include young adults as the control group [32,33]. However, the characteristics of declining function in elderly people could be observed more clearly in comparisons with children, whose function has not matured. Therefore, children were included in the participant group in the current study.

The first purpose of the current study was to determine whether equilibrium was associated with cognitive function, by examining the coloration of vividness and controllability in the imaginary body movement test and body schema, using the body sway index during the one-leg standing test. The second purpose of the study was to elucidate the characteristics of equilibrium in elderly people when compared to that of children and college students. 


\section{Material and Methods}

\section{Participants}

The study included 139 healthy individuals. Adult participants and children's primary caregivers provided written informed consent in accordance with the requirements for local ethical approval. Participants were assigned to one of three age groups: 41 children (5-6 years; 19 boys, 22 girls), 30 students (18-21 years; 17 men, 13 women), and 68 older adults (60-86 years; 32 men, 36 women). Participants enrolled in the study voluntarily after receiving oral explanations regarding the study purpose and method, any expected discomfort, and their freedom to discontinue participation. Upon completion of the study, the adult participants were debriefed about the meaning of their task scores. All experiments were conducted in compliance with the study protocol, which was approved by the research ethics committee at Shiga University, Japan (Number 2015-3).

The children and students were recruited from a public kindergarten and a university, respectively. The older adults were recruited from a job center that offers older retirees employment. None of the participants had been diagnosed with any specific neurological or metabolic disorders that could have influenced their performance in the experimental tasks, and all had normal or corrected-to-normal vision. Upon completion of the experiment, the students and older adults were interviewed about their strategies in the imaginary body movement test, but the children were not, because they were unable to describe their strategies appropriately. In total, 96 of the 98 interviewees reported imaging body movement. The remaining two older adults reported using a perceptual updating strategy and were excluded from the analysis.

\section{Design}

In the posturography, the participants were asked to stand on one leg with their eyes open. As the one-leg standing test had demonstrated sufficient test-retest reliability, only one measurement was taken [34]. The imaginary body movement test involved a hide-and-seek video game developed by Watanabe, in which participants attempt to locate a child hiding in a house [27]. One experimental session contained nine questions at different rotation angles of the house. Participants repeated the video game task in two conditions: sitting in an armchair with the non-dominant arm bound to an armrest (restrained condition) and standing on an unstable air mat, which yielded large somatic sensations (unstable condition). Participants completed two experimental sessions in each condition. The condition order was counterbalanced within each age group.

\section{Apparatus}

A posturograph (T.K.K.5810) manufactured by Takei Scientific Instruments Co., Ltd., Japan was used to measure the barycentric track. Sampling frequency was set at $20 \mathrm{~Hz}$ using the accessory software for the posturograph, which was installed on a laptop (SONY VAIO VJS1111) connected to the posturograph, and the coordinate for the center of gravity was measured after removing noise by taking the mean value for 10 movement data. The total sway path length $(\mathrm{mm})$, which is the sum of the distance between center of gravity coordinates; length per second $(\mathrm{mm} / \mathrm{s})$, which refers to the length of the track per 1 $\mathrm{s}$; and the outer area $\left(\mathrm{mm}^{2}\right)$, which is a multangular area that includes all center of gravity coordinates and minimal boundary lengths, were recorded. The apparatus used in a study conducted by Watanabe were used in the imaginary body movement test and included a video game machine (XaviX PORT; SSD Co., Ltd., Japan), two different sizes of armchair for use in the restrained condition, and a polyvinyl chloride air mat for use in the unstable condition [27].

\section{Procedure}

In the one-leg standing test, a trial was performed before measurement to confirm whether one-leg standing was feasible for 15 s. If the tester determined that 15-s one-leg standing was too difficult, the duration was shortened and participants were required to stand on one leg with their eyes open for either 10 or $5 \mathrm{~s}$ according to ability. Data for four elderly adults (two men) who retained this position for $<5 \mathrm{~s}$ were excluded from the analysis. No children or college students were excluded from the analysis or required shortened standing time. The pivoting foot was placed at the center of the posturograph, and the other foot was raised upon the receipt of a signal from the tester. Participants were asked to stare at a mark on the wall at eye level directly ahead and maintain as stable a posture as possible. Once the tester confirmed that the participants were standing on one leg, the timer in the posturography software was started and measured the movement of the gravity center automatically for the predetermined number of seconds.

The procedure in the imaginary body movement test was that used by Watanabe [27]. In the restrained condition, participants sat with their backs resting against the back of an armchair. In the unstable condition, they stood on the air mat without shoes. After completing one condition, participants were introduced to the next condition rapidly. The participants were required to identify a window in the house via which to find the hiding child. At the beginning of the game, two questions were presented at the $0^{\circ}$ position in the unrotated house, followed by seven questions at other positions with in the rotated house. The angles of rotation in the picture plane (i.e., $45^{\circ}, 90^{\circ}, 135^{\circ}$, $180^{\circ}, 225^{\circ}, 270^{\circ}$, and $315^{\circ}$ counter-clockwise) were randomized. Response time was defined as the time that elapsed between the "Start" signal and the point at which the participants identified a window in the house. The player's response times for each rotation angle were automatically recorded by the video-game controller.

\section{Indices}

In the one-leg standing test, total sway path length was divided by the outer area, and the length per area $\left(\mathrm{mm} / \mathrm{mm}^{2}\right)$ was calculated. Length per second and length per area were adopted as indices, as they can be compared between age groups regardless of standing time. The speed represented by length per second is considered a sensitive index of the general equilibrium and increases with age during adulthood [19]. Length per area, which represented path density, indicated that the efficiency of posture control decreased with age during adulthood, in contrast to length per second [19].

The calculation of the index of imaginary body movement followed that described by Watanabe [27]. Values over 2.5 SD from each mean were considered outliers, which allowed for $99 \%$ confidence intervals for response times. Data for 4 children and 2 older adults with at least one outlier in any condition and session were excluded from the subsequent analysis, because such outliers indicated that the participants' attention was captured temporarily by something extrinsic to the task. The mean response times for the eight locations from $0^{\circ}$ to $315^{\circ}$ displayed a hill-like curve that peaked at approximately $180^{\circ}$ in each age group and condition. Rotation angles that were 
symmetrical to the participants' median line were at equal distances from the participants (e.g., $90^{\circ}$ and $270^{\circ}$ ). Therefore, data corresponding to rotation angles of $>180^{\circ}$ and $<180^{\circ}$ were included.

Linear formulas were calculated using the least squares method for each participant; condition with degrees of rotation between $45^{\circ}$ and $180^{\circ}$ was included as an explanatory variable, and response time was included as a response variable. In the formula $y=a x+b(x$ : degrees of rotation, y: response time), the gradient represents the theoretical time per degree for imaginary body movement. Each gradient was multiplied by 180 to represent the theoretical response time for rotation of the representational self mentally to the $180^{\circ}$ position. Conditions based on this gradient-based index were labeled response time in restrained condition (RT-R) and response time in unstable condition (RT-U).

When the RT-R or RT-U became negative, the data became ineffective, as it was possible that other cognitive strategies than the expected imaginary body movement was used or attention was extremely scattered during the test. When both trials were effective in either the stable (restrained) or unstable condition, the value of the second trial was adopted, and when one of the two trials was ineffective, the value of the effective trial was adopted; when neither was effective, the participant was excluded from the analysis. Data for three children and two elderly adults were excluded from the analysis. Moreover, when the number of correct responses was $\leq 6$ out of 9 , there was concern that task comprehension had been inefficient or the participant had not focused on the task. Data for two children and two elderly adults who provided $<6$ correct responses were excluded from the analysis. Furthermore, nine children and 12 elderly adults were excluded from the analysis, as follows: four elderly adults were excluded from the standing test, and two elderly adults were excluded because of their responses in the interview conducted after the experiment examining imaginary body movement. The participants for whom data were analyzed included 32 children (5-6 years; 14 boys, 18 girls), 30 college students (18-21 years; 17 men, 13 women), and 56 elderly adults (62-86 years; 25 men, 31 women).

\section{Statistical analysis}

The retention time for the one-leg standing test with eyes open was $15 \mathrm{~s}, 10 \mathrm{~s}$, or $5 \mathrm{~s}$. ANOVAS were performed, with retention time as the independent variable and length per second or length per area as the dependent variable, to examine the relationship between retention time and the movement of the gravity center in the one-leg standing test, in which retention time varied between the elderly population groups (Table 1).

\begin{tabular}{|c|c|c|}
\hline Duration levels & Length per s† & Length per area†† \\
\hline 5 seconds $(N=17)$ & $38.66 \pm 20.57^{*}$ & $0.37 \pm .19^{* *}$ \\
\hline 10 seconds $(\mathrm{N}=8)$ & $32.92 \pm 13.21^{*}$ & $0.47 \pm .20^{\star *}$ \\
\hline 15 seconds $(N=31)$ & $25.33 \pm 6.15^{\star}$ & $0.57 \pm .20^{\star \star}$ \\
\hline
\end{tabular}

Table 1: Means and standard deviations for the indexes of one-leg standing tests in three duration levels.

The elderly adults were divided into two groups according to standing time (older adults L group: $5 \mathrm{~s}$ or $10 \mathrm{~s}, \mathrm{n}=31 ; 62-86$ years; 13 men, 18 women; older adults $\mathrm{H}$ group: $15 \mathrm{~s}, \mathrm{n}=25$; 63-79 years; 12 men,
13 women). The subsequent analysis was performed with four age groups including these two groups. Means and standard deviations were calculated for length per second or length per area in the one-leg standing test according to age group (Table 2). A one-way ANOVA was performed with age group as the independent variable.

\begin{tabular}{|c|c|c|c|}
\hline & Mean \pm SD & Minimum & Maximum \\
\hline \multicolumn{3}{|l|}{ Children } & \\
\hline Length per s† & $18.65 \pm 6.60^{* *}$ & 9.1 & 33.8 \\
\hline Length per area†† & $.67 \pm .33^{\star *}$ & 0.24 & 1.45 \\
\hline \multicolumn{4}{|l|}{ Students } \\
\hline Length per s $\dagger$ & $28.39 \pm 21.13$ & 9.8 & 94.9 \\
\hline Length per area†† & $1.30 \pm .44^{* *}$ & 0.56 & 2.45 \\
\hline \multicolumn{4}{|l|}{ Older adults $\mathrm{H}$} \\
\hline Length per s† & $25.24 \pm 5.87^{\star *}$ & 14.5 & 39.8 \\
\hline Length per area† $\dagger$ & $.57 \pm .20^{* *}$ & 0.26 & 1 \\
\hline \multicolumn{4}{|l|}{ Older adults $\mathrm{L}$} \\
\hline Length per s† & $35.69 \pm 18.82^{\star *}$ & 12.7 & 93.5 \\
\hline Length per area†† & $.40 \pm .20^{\star \star}$ & 0.05 & 0.9 \\
\hline
\end{tabular}

Table 2: Descriptive statistics for one-leg standing tests.

Discriminant analysis was performed with the two age groups combined as the standard variable, and two indices in the one-leg standing test (length per second and length per area) were standardized to form an explanatory variable. The backward stepwise procedure in which AIC was minimized for selecting the variables to take in was used and the discriminant function was determined on the condition that the proportion of correct classifications using the remaining variables was $\geq 75 \%$. The determinant function fulfilled this condition when the child group and college group; college group, older adults L group, and older adults $\mathrm{H}$ group; and child group and older adults $\mathrm{L}$ group were combined to form standard variables.

Pearson's product-moment correlation coefficients for the association between length per second and length per area were calculated according to age group. In addition, Pearson's productmoment correlation coefficients were calculated for the association between total sway path length and the outer area were calculated according to age group. Means and standard variables for the RT-R and RT-U in imaginary body movement were calculated for each age group (Table 3). A one-way ANOVA was performed with age group as the independent variable, and Scheffe's method was used to perform multiple comparisons.

The imaginary body movement used in the current study is also used in clinical medicine. When determining whether brain dysfunction is present, the cut-off value corresponding to the RT-R is $1,800 \mathrm{~ms}$ [35]. Moreover, young adults, such as college students, have been shown to react within $800 \mathrm{~ms}$ [30]. Based on this finding, participants were categorized into three functional groups: the healthy group ( $\mathrm{N}=61 ; 26 \mathrm{men}, 35$ women) in which the RT-R was $<800 \mathrm{~ms}$; the borderline group ( $\mathrm{N}=37 ; 18$ men, 19 women), in which the RT-R was 
Citation: Watanabe M (2018) Does the Controllability of the Body Schema Predict Equilibrium in Elderly People? Characteristics of Relationships from a Lifelong Development Perspective. J Aging Sci 6: 191. doi:10.4172/2329-8847.1000191

Page 5 of 9

800-1,800 ms; and the disability group ( $\mathrm{N}=20 ; 12 \mathrm{men}, 8$ women), in which the RT-R was $>1,800$ ms with a risk of function decline.

\begin{tabular}{|c|c|c|c|}
\hline & Mean \pm SD & Minimum & Maximum \\
\hline \multicolumn{4}{|l|}{ Children } \\
\hline Stable & $1501.08 \pm 1012.41^{\text {** }}$ & 301.81 & 3962.69 \\
\hline Unstable & $1090.99 \pm 938.48^{* *}$ & 138 & 3781.35 \\
\hline \multicolumn{4}{|l|}{ Students } \\
\hline Stable & $495.59 \pm 490.41^{\star \star}$ & 3.95 & 1724.15 \\
\hline Unstable & $357.87 \pm 423.77^{\star \star}$ & 1.68 & 1694.92 \\
\hline \multicolumn{4}{|c|}{ Older adults $\mathrm{H}$} \\
\hline Stable & $937.87 \pm 729.20$ & 162.46 & 3575.15 \\
\hline Unstable & $803.69 \pm 425.70$ & 81 & 1809.46 \\
\hline \multicolumn{4}{|c|}{ Older adults $L$} \\
\hline Stable & $1554.87 \pm 1456.53^{\star *}$ & 100.39 & 6308.85 \\
\hline Unstable & $965.18 \pm 565.26^{* *}$ & 109.77 & 1882.77 \\
\hline \multicolumn{4}{|l|}{${ }^{* *}: p<.01$} \\
\hline
\end{tabular}

Table 3: Descriptive statistics for one-leg standing tests.

An independent chi-square test and a residue analysis were performed, with a cross table for four age groups and three function groups (Table 4). Pearson's product-moment correlation coefficients were calculated for associations between the two indices (length per second and length per area) in the one-leg standing test and the two indices (the RT-R and RT-U) in the imaginary body movement test were calculated according to age group (Table 5). Pearson's productmoment correlation coefficient was also calculated for the association between total sway path length and the outer area and provided a basic calculation via which to obtain length per second and length per area with RT-U.

\begin{tabular}{|l|l|l|l|}
\hline & Normal & $\begin{array}{l}\text { Borderlin } \\
\text { e }\end{array}$ & Disability \\
\hline Children & 14 & 6 & 12 \\
\hline & -1.054 & -1.8 & $3.630 \triangle^{* *}$ \\
\hline Students & 21 & 9 & 0 \\
\hline & $2.323 \triangle^{*}$ & -0.185 & $-2.865 \nabla^{* *}$ \\
\hline Older adults H & 17 & 11 & 3 \\
\hline & 0.408 & 0.577 & -1.257 \\
\hline & 9 & 11 & 5 \\
\hline Older adults L & -1.769 & 1535 & $0.458^{*}$ \\
\hline & 14 & 6 & 12 \\
\hline
\end{tabular}

Upper line: frequency; Lower line: adjusted standardized residual; $\Delta$ : significantly more; $\mathbf{\nabla}$ : significantly less; *:p<.05; **:p<.01

Table 4: Frequencies and adjusted standardized residual between age groups and function groups.

\begin{tabular}{|l|l|l|}
\hline \multicolumn{2}{|l|}{ RT-R } & RT-U \\
\hline Children & 0.203 & $.559^{* *}$ \\
\hline Length per s & -0.199 & $-.351^{*}$ \\
\hline Length per area & $-.496^{* *}$ & $-.403^{*}$ \\
\hline Students & $-.558^{* *}$ & $-.446^{* *}$ \\
\hline Length per s & \multicolumn{2}{|l|}{} \\
\hline Length per area & 0.214 & 0.19 \\
\hline Older adults H & -0.049 & 0.026 \\
\hline Length per s & \multicolumn{2}{|l|}{} \\
\hline Length per area & 0.26 & $-.358+$ \\
\hline Older adults L & -0.032 & $.466^{*}$ \\
\hline Length per s & \multicolumn{2}{|l}{} \\
\hline Length per area & \multicolumn{2}{|l|}{} \\
\hline$+:$ p $<.10 ; *^{*}$ p $<.05 ; * *: p<.01$ &
\end{tabular}

Table 5: Pearson product-moment correlation coefficients between one-leg standing test with eyes open and response times of the imaginary body movement test.

\section{Results}

\section{Characteristics of the results of the one-leg standing test}

Significant main effects were observed for both length per second $\left(\mathrm{F}=4.27, \mathrm{df}=2.53 ; \mathrm{p}=.00191 ; \eta^{2}=.14\right)$, and length per area $(\mathrm{F}=5.20$; $\mathrm{df}=2,53 ; \mathrm{p}=.0087 ; \eta^{2}=.16$ ). In the multiple comparison using the Scheffe's method, participants who were able to stand on one leg for 15 $s$ exhibited significantly shorter length per second $(\mathrm{F}=4.00 ; \mathrm{df}=2,53$; $\mathrm{p}=.0242 ; \mathrm{r}=.27)$ and significantly longer length per area $(\mathrm{F}=5.14$; $\mathrm{df}=2,53 ; \mathrm{p}=.0091 ; \mathrm{r}=.30$ ) relative to that of participants who were able to stand on one leg for $5 \mathrm{~s}$.

The results of the one-way ANOVA with age group as the independent variable showed significant main effects for length per second $\left(\mathrm{F}=6.53 ; \mathrm{df}=3,114 ; \mathrm{p},<.001 ; \eta^{2}=.15\right)$ and length per area $\left(\mathrm{F}=44.77 ; \mathrm{df}=3,114 ; \mathrm{p}<.001 ; \eta^{2}=.54\right)$. Multiple comparison using Scheffe's method showed that length per second in children was significantly shorter relative to that observed in the older adult $\mathrm{L}$ group $(\mathrm{F}=6.27 ; \mathrm{df}=3,114 ; \mathrm{p}<.001 ; \mathrm{r}=.23)$, and length per area in college students was significantly longer relative to that observed in children $(\mathrm{F}=20.59 ; \mathrm{df}=3,114 ; \mathrm{p}<.001 ; \mathrm{r}=.39)$, the older adult $\mathrm{H}$ group $(\mathrm{F}=27.57$; $\mathrm{df}=3,114 ; \mathrm{p}<.001 ; \mathrm{r}=.44)$, and the older adult $\mathrm{L}$ group $(\mathrm{F}=36.84$, $\mathrm{df}=3,114 ; \mathrm{p}<.001 ; \mathrm{r}=.49$ ). In addition, length per area in children was significantly longer relative to that observed in the older adult $\mathrm{L}$ group $(\mathrm{F}=3.38 ; \mathrm{df}=3,114 ; \mathrm{p}=.021 ; \mathrm{r}=.17)$. The results of the discriminant 
analysis showed that length per area was effective in separating college students from children and elderly adults, and length per second was effective in separating children from the older adults $\mathrm{L}$ group.

Pearson's product-moment correlation coefficients showed a moderate to strong correlation between length per second and length per area in children $(\mathrm{r}=-.617, \mathrm{t}=4.29, \mathrm{df}=30, \mathrm{p}<.001)$, college students $(\mathrm{r}=.467, \mathrm{t}=2.80, \mathrm{df}=28, \mathrm{p}=.009)$, the older adults $\mathrm{H}$ group $(\mathrm{r}=-.584$, $\mathrm{t}=3.88, \mathrm{df}=29, \mathrm{p}<.001)$, and the older adults $\mathrm{L}$ group $(\mathrm{r}=-.751, \mathrm{t}=5.46$, $\mathrm{df}=23, \mathrm{p}<.001)$, a negative correlation between these variables in children and elderly adults, and a positive correlation between these variables in college students.

In addition, Pearson's product-moment correlation coefficients showed a medium to strong correlation between total sway path length and the outer area in children $(\mathrm{r}=.912, \mathrm{t}=12.15, \mathrm{df}=30, \mathrm{p}<.001)$, college students $(\mathrm{r}=.851, \mathrm{t}=8.60, \mathrm{df}=28, \mathrm{p}<.001)$, the older adult $\mathrm{H}$ group $(\mathrm{r}=$. 753, $\mathrm{t}=6.17, \mathrm{df}=29, \mathrm{p}=.002)$, and the older adult $\mathrm{L}$ group $(\mathrm{r}=.582$, $\mathrm{t}=3.43, \mathrm{df}=23, \mathrm{p}<.001)$.

\section{Characteristics of the imaginary body movement}

The results of the one-way ANOVA with age group as an independent variable showed significant main effects for both the RT$\mathrm{R}\left(\mathrm{F}=7.66 ; \mathrm{df}=3,114 ; \mathrm{p}<.001, \eta^{2}=.17\right)$ and $\mathrm{RT}-\mathrm{U}(\mathrm{F}=7.46 ; \mathrm{df}=3,114, \mathrm{p}<$. $\left.001, \eta^{2}=.16\right)$. In the multiple comparison using Scheffe's method, college students showed a significantly smaller value relative to those of children $(\mathrm{F}=5.43 ; \mathrm{df}=3,114 ; \mathrm{p}=.002 ; \mathrm{r}=.21)$ and the older adult $\mathrm{L}$ group $(\mathrm{F}=5.31, \mathrm{df}=3,114 ; \mathrm{p}=.002, \mathrm{r}=.21)$ in the RT-R, and college students showed a significantly smaller value in the RT-U relative to that observed in children $(\mathrm{F}=6.70 ; \mathrm{df}=3,114 ; \mathrm{p}<.001 ; \mathrm{r}=.24)$ and the older adult $\mathrm{L}$ group $(\mathrm{F}=4.05 ; \mathrm{df}=3,114 ; \mathrm{p}=.009 ; \mathrm{r}=.19)$.

The results of the independent chi-square test and residue analysis showed a significant bias $\left(\chi^{2}=20.83, \mathrm{df}=6, \mathrm{p}=.002\right.$, Cramer's $\left.\mathrm{V}=.30\right)$; a significantly high proportion of the children were in the disability group, a significantly high proportion of college students were in the healthy group, and a significantly low proportion of the college students were in the disability group. Moreover, of the six elderly adults, who were excluded from the analysis because they could perform the one-leg standing with eyes open for $<5 \mathrm{~s}$, three were in the boundary group, and three were in the disability group.

\section{Relationship between the one-leg standing test and the imaginary body movement test}

Pearson's product-moment correlation coefficients showed a moderate positive correlation between length per second and the RT-U and a slightly negative correlation between length per area and the RT$\mathrm{U}$ in children. The former finding indicates that imaginary body movement declined as gravity movement increased in the unstable condition. The latter indicates that as density gravity movement increased, the speed of the imaginary body movement increased in the unstable condition. In college students, a moderate negative correlation was observed in all combinations of the indices for the oneleg standing test and the imaginary body movement.

This indicates that faster gravity movement and higher density were associated with faster imaginary body movement in both the stable and unstable conditions. A moderate positive correlation was observed between length per area and the RT-U, and a weak but significant negative correlation was observed between length per second and the RT-U. The former indicates that higher density of the gravity movement was associated with slower imaginary body movement in the unstable condition. The latter indicates that faster gravity movement was associated with increased imaginary body movement in an unstable condition. No significant correlations were observed in the older adult $\mathrm{H}$ group.

Pearson's product-moment correlation coefficient for the associations between RT-U and total sway path length and the outer area, which is the basic calculation via which to obtain length per second and length per area, showed moderate positive correlations between total sway path length and RT- $\mathrm{U}$ in children $(\mathrm{r}=.453, \mathrm{t}=2.78$, $\mathrm{df}=30, \mathrm{p}=.009)$ and the outer area and the RT-U $(\mathrm{r}=.455, \mathrm{t}=2.80, \mathrm{df}=30$, $\mathrm{p}=.009$ ). In the college student group, a medium level of negative correlation was obtained with the total sway path length $(r=-.572$, $\mathrm{t}=3.69, \mathrm{df}=28, \mathrm{p}<.001)$ and a slight negative correlation with the outer area $(\mathrm{r}=-.432, \mathrm{t}=2.54, \mathrm{df}=28, \mathrm{p}=.017)$. No significant correlation was shown in either of the two elderly groups.

\section{Discussion}

The analysis of the characteristics of the one-leg standing test showed that length per second increased from childhood to adulthood and continued to do in the older adult L group when standing ability deteriorated, and length per area increased from childhood to adulthood but declined in older adulthood. In the discriminant analysis, length per area discriminated between college students and the other three groups; college students exhibited longer length per area relative to that observed in the other groups, and length per second discriminated between children and the older adult L group; children exhibited shorter length per second relative to that observed in the older adult $\mathrm{L}$ group. This result is consistent with conventional knowledge indicating that length per second increased with aging during all stages of adulthood but differs from the results reported by Rival, Ceyte, and Olivier's who concluded that length per second decreased linearly from childhood to young adulthood [31].

In contrast, the result regarding length per area supported the general opinion that it peaks in young adulthood and follows a mountain shape. Based on this assumption, the characteristics of postural control in each age group during one-leg standing can be summarized as follows. In children, the center of weight swung slowly within a comparatively wide range; therefore, both length per second and length per area were short. In college students, minute adjustments were made within a small range; therefore, both length per second and length per area were long. Conversely, in the two elderly groups, the center of weight moved rapidly within a wide range; therefore, while length per second was long, length per area was short.

The analysis of the characteristics of the imaginary body movement indicated that the mean observed for elderly people in the stable condition was almost identical to the standardized mean, as observed by Watanabe and Takamatsu, who reported $1,048.5 \mathrm{~ms}$, and Watanabe, who reported $1,391.3 \mathrm{~ms}$ [26,30]. In addition, for both the RT-R and RT-U, achievement in college students was superior to that of children and elderly people. In particular, with respect to RT-R, numerous children were categorized into the disability group, in which ability is largely inferior, and numerous elderly people were categorized in the boundary group, in which ability had declined somewhat. These results suggest that imaginary body movement develops rapidly from childhood to young adulthood and decreases during old age. This corresponds with Watanabe and Takamatsu's findings [30]. Therefore, 
the measurement values obtained in the study were verified as appropriate.

In view of the above, we analyzed the relationship between the standing test and imaginary body movement test. Indices of equilibrium and cognitive function were observed mainly in college students, indicating that there is indeed an association between these functions. However, moderate correlations were observed in some elderly people. Furthermore, the relationship between equilibrium and cognitive function differed between children and elderly adults. In children, RT-U was positively correlated with length per second and negatively correlated with length per area. However, in the older adult L group, RT-U was negatively correlated with length per second and positively correlated with length per area. In view of these findings, we presumed the following concerning the characteristics of equilibrium in elderly people.

In college students, as the values for the RT-R and RT-U were low (i.e., the functions are superior) and those for length per second and length per area were high, the body schema was controlled appropriately because of the high controllability function, and the center of weight was retained at the small base of support standing on one leg. Moreover, as the RT-U was negatively correlated with both length per second and length per area, higher controllability levels (i.e., low values for the RT-U is) were associated with rapid movement of the center of weight (increasing with length per second). However, as the RT-U was weakly negatively correlated with the outer area, it appeared that greater controllability was associated with wider shifts in the center. To summarize, in young adulthood, participants used a method via which high-speed body sway retained the center of weight within the smallest range possible, and speed was profoundly correlated with controllability.

In contrast, as children's controllability was inferior to that of adults, the participants could have been poor at changing their body schemas rapidly. Therefore, it would have taken time to recover from the decline in posture. This is congruent with the opinion that the center of weight moves slowly within a comparatively wide range, reflecting the characteristics of postural control in childhood. Moreover, with higher controllability (the RT-U value decreased), length per second was reduced and length per area increased. As the RT-U was moderately positively correlated with the outer area, when controllability increased with development, body sway could be retained within a smaller range. Moreover, as the RT-U was moderately positively correlated with total sway path length, the latter also decreased with the development of controllability and reduction of the outer area. Furthermore, the positive correlation between the RT-U and length per second also indicated that the speed of body sway decreased. Therefore, if the outer area, total sway path length, and speed of sway decreased simultaneously, length per area, which is the value of the total sway path length divided by the outer area, should not have changed vertically, and this seemingly contradicts the negative correlation between RT-U and length per area.

However, considering that the reduction of the outer area occurred more rapidly relative to that of total sway path length and speed of sway, a consistent explanation is possible. In other words, with the development of postural control in childhood, the area of body sway is minimized mainly via the improvement of controllability. To some extent, this could be associated with the decrease in track length and reduction of sway speed. This finding corresponds to those reported by Rival, Ceyte, and Olivier, which showed that the area of sway decreased in a linear manner from the age of 6 years [31]. In addition, length per second in college students was significantly longer relative to that observed in children. Therefore, the reductions in the area of body sway and sway speed occur from the age of 6 years. However, sway speed later increases and high-speed body sway will occur within a small range.

What are the characteristics of equilibrium and cognitive function in elderly people when compared with children and college students? The two groups of elderly people with higher RT-U values, relative to that of college students, demonstrated that controllability decreased. Furthermore, in the older adult L group, higher levels of controllability (the RT-U value decreased) were associated with longer length per second and shorter length per area. However, this relationship did not demonstrate a direct correlation between controllability and equilibrium function. The reason for this is that, with two groups of elderly people, there were no significant correlations between RT-U and total sway path length or the outer area. Moreover, in the older adult $\mathrm{H}$ group, there was no relationship between RT-U and equilibrium. Therefore, in the elderly people, the reduction of controllability did not appear to be the direct reason for changes in body sway in the one-leg standing test.

Aside from controllability, the factor that exerted the greatest influence on standing ability was reduced function in motor organs such as muscles and joints [8]. Even in cases in which elderly people are expected to maintain cognitive control originating from controllability to the same extent as young adults, standing could become unstable because of the deterioration of the motor organs. In addition, lower levels of controllability (with higher RT-U values) are associated with the need for longer periods to return to a normal posture, and the center of weight shifts rapidly, for a long period, and within a broad range; consequently, length per second is likely to increase. Distortion of the standing posture, caused by the deterioration of the motor organs, could easily generate a considerable distortion of balance. In fact, when multiple comparisons were performed for the total sway path length and the outer area using the Bonferroni method for the three comparable groups (i.e., children, college students, and the older adult $\mathrm{H}$ group), the outer area in the older adult $\mathrm{H}$ group was larger relative to those observed for children and college students, but the total sway path length in the older adult $\mathrm{H}$ group was middle between children and college students.

As demonstrated above, when the outer area was extended further than the total sway path length, the values for length per area declined. These influential factors, which originated from the decline of motor organs, covered the relationship between equilibrium and controllability in the older adult $\mathrm{H}$ group and canceled out the correlation. However, in the older adult L group, whose motor organ decline was advanced, the effect was shown as a correlation. In this way, the decline of controllability does deteriorate in elderly people; however, this does not mean that it returns to the condition in which the center of the weight sways slowly within a wide range, as observed in childhood. Rather, elderly people repeat body sways as rapidly as possible, similar to college students, to maintain a standing posture. However, the deterioration of the motor organs could cause instability in the standing position, and movement of the gravity center occurs vigorously in the recovery of their posture. To summarize, equilibrium in elderly people has not changed with respect to strategies for postural control, which continue from young adulthood; however, the dynamic balance decreases slowly with a gradual reduction in the controllability of the body schema, and balance is lost because of the decline of the motor organs that occur in parallel. Patterns to cope with these 
multiple functions exist. The reason why Demnitz et al. reported that there was no relationship between equilibrium and cognitive function in the meta-analysis was that these complicated factors are unique to elderly people [11].

Moreover, there was no relationship between vividness and equilibrium in children or the two elderly groups. However, there was a negative correlation between vividness and length per second or length per area in college students; therefore, there is no doubt that vividness was related to equilibrium. The strong influenced of immaturity in controllability in children and the decline in motor organs experienced by elderly people could explain the effects of vividness in these groups. Changes in the vividness of the body schema in development from childhood to early old age are small [28]. Therefore, the vividness is not a strong predictor of standing ability in the elderly.

In generics and physical therapy, sarcopenia [36], which refers to loss of muscle mass and power as factors that are strongly related to fitness for performance and frailty in elderly people, has recently gained attention, and examination of its relationship with standing ability has increased [37]. However, it should be noted that there many factors decrease standing retention time and stability in elderly people, and of all these factors, equilibrium is the most influential predictor of repeated falling [2]. The controllability of the body schema, which verifies a connection with equilibrium, is known to emerge in early childhood and continue in developmental change until old age $[38,39]$. Further, research using noninvasive brain imaging technology has shown that the function is performed within the motor-related brain areas such as the left precuneus and premotor area and left supplementary motor area $[40,41]$, and these brain functions do not deteriorate in a single unique manner in elderly people [42]. Moreover, a previous study showed that these functions are closely related to physical recovery rates in the acute period of rehabilitation following a stroke [35]. Diseases affecting the motor organs and decreasing muscle mass are important issues to consider in preventing falls in elderly people. It is necessary to proceed with research with careful attention to controllability of the body schema related to determination of the coordination of physical behavior, which is critical to retaining dynamic balance and deeply related to developmental psychology, neuroscience, and clinical medicine.

This study showed that controllability of the body schema, which is a cognitive function, is strongly connected to equilibrium in the context of life-long development. This finding suggested that reduced controllability in elderly people could explain part of the decline in their equilibrium, and this change in elderly people is a product of multiple factors, which differ from the developmental changes that occur from childhood to adulthood. If this area of research is advanced, it will be possible to extrapolate age-related reduction of standing ability to cognitive functions such as the controllability of the body schema. Moreover, in rehabilitation and function retention training, this can be related to effective program development, which could be applied simultaneously for motor and cognitive function. In this respect, the findings of this study could mark the beginning of a new research are contributing to health maintenance in elderly people.

\section{Acknowledgement}

This work was supported by JSPS KAKENHI Grant Numbers JP $15 \mathrm{~K} 04060$

\section{References}

1. Nevitt MC (1997) Falls in the elderly: Risk factors and prevention. In: Masdeu JC, Sudarsky L, Wolfson L (eds.) Gait disorders of aging: Falls and therapeutic strategies. Lippincott-Raven, Philadelphia, USA. pp. 13-36.

2. Stalenhoef AP, Diederiks JP, Knottnerus AJ, Kester DA, Crebolder FH (2002) A risk model for the prediction of recurrent falls in communitydwelling elderly: a prospective cohort study. J Clin Epidemiol 55: 1088-1094.

3. Murphy J, Isaacs B (1982) The post-fall syndrome. A study of 36 elderly patients. Gerontology 28: 265-270.

4. Binda SM, Culham EG, Brouwer B (2003) Balance, muscle strength, and fear of falling in older adults. Exp Aging Res 29: 205-219.

5. Rubenstein LZ, Josephson KZ (2002) The epidemiology of falls and syncope. Clin Geriatr Med 18: 141-158.

6. Hauck JL, Carpenter GM, Frank SJ (2008) Task-specific measures of balance efficacy, anxiety, and stability and their relationship to clinical balance performance. Gait Posture 27: 676-82.

7. Thomas IJ, Lane VJ (2005) A pilot study to explore the predictive validity of 4 measures of fall risk in frail elderly patients. Arch Phys Med Rehabil 86: $1636-1640$

8. Luria R, Meiran N (2005) Increased control demand results in serial processing: evidence from dual-task performance. Psychol Sci 16: 833-840.

9. Tabara Y, Okada Y, Ohara M, Uetani E, Kido T, et al. (2015) Association of postural instability with asymptomatic cerebrovascular damage and cognitive decline: The Japan Shimanami health promoting program study. Stroke 46: 16-22.

10. Fujita H, Kasubuchi K, Wakata S, Hiyamizu M, Morioka S (2016) Role of the frontal cortex in standing postural sway tasks while dual tasking: a functional near-infrared spectroscopy study examining working memory capacity. BioMed research international: Article ID 7053867.

11. Demnitz N, Esser P, Dawes H, Valkanova V, Johansen-Berg H, et al. (2016) Systematic review and meta-analysis of cross-sectional studies examining the relationship between mobility and cognition in healthy older adults. Gait Posture 50: 164-174.

12. Dichgans J, Brandt T (1978) Visual-vestibular interactions: effects on selfmotion perception and postural control. In: Held R, Leibowitz H, Teuber HL (eds.) Handbook of Sensory Physiology, Perception (8th edn.). Springer, Berlin, Germany pp.755-804.

13. Head H (1920) Studies in Neurology (2th edn.). Oxford University Press, London, UK.

14. Gallagher S, Cole J (1995) Body schema and body image in a deafferented subject. J Mind Behav 16: 369-390.

15. Browne J, O'Hare N, O'Hare G, Finn A, Colin J (2002) Clinical assessment of the quantitative posturography system. Physiotherapy 88 : 217-223.

16. Bohannon R (2006) Single Limb Stance times a descriptive Meta-Analysis of data from individuals at least 60 years of age. Top Geriatr Rehabil 22: 70-77.

17. Springer BA, Marin R, Cyhan T, Roberts H, Gill NW (2007) Normative values for the unipedal stance test with eyes open and closed. J Geriatr Phys Ther 30: 8-15.

18. Michikawa T, Nishiwaki Y, Takebayashi T, Toyama Y (2009) One-leg standing test for elderly populations. J Orthop Sci 14: 675-685.

19. Fujita T, Nakamura S, Ohue M, Fujii Y, Miyauchi A, et al. (2005) Effect of age on body sway assessed by computerized posturography. J Bone Miner Res 23: 152-156.

20. Fuchioka S, Iwata A, Higuchi Y, Miyake M, Kanda S, et al. (2015) The forward velocity of the center of pressure in the midfoot is a major predictor of gait speed in older adults. Int J Geronttol 9: 119-122.

21. Isaac A, Marks DF, Russell DG (1986) An instrument for assessing imagery of movement: The Vividness of Movement Imagery Questionnaire (VMIQ). J Mental Imagery 10: 23-30. 
Citation: Watanabe M (2018) Does the Controllability of the Body Schema Predict Equilibrium in Elderly People? Characteristics of Relationships from a Lifelong Development Perspective. J Aging Sci 6: 191. doi:10.4172/2329-8847.1000191

Page 9 of 9

22. Naito E (1994) Controllability of motor imagery and transformation of visual imagery. Percept Mot Skills 78: 479-87.

23. Gawron W, Pospiech L, Orendorz-Fraczkowska K, Noczynska A (2002) The influence of metabolic disturbances present in diabetes mellitus type I on vestibule-spinal reflexes in children and young adults. Polish Otolaryngology 56: 451-457.

24. Lemos T, Souza NS, Horsczaruk CHR, Nogueira-Campos AA, De Oliveira LS, et al. (2014) Motor imagery modulation of body sway is taskdependent and relies on imagery ability. Front Hum Neurosci 8: 1-9.

25. Watanabe $M$ (2011) Distinctive features of spatial perspective-taking in the elderly. Int J Aging Hum Dev 72: 225-241.

26. Piaget J, Inhelder B (1947) La représentation de l'espace chez l'enfant. Presses Universitaires de France, Paris, France.

27. Watanabe M (2016) Developmental changes in the embodied self of spatial perspective taking. Br J Dev Psycho 34: 212-225.

28. Schott N (2012) Age-related differences in motor imagery: working memory as a mediator. Exp Aging Res 38: 559-583.

29. Saimpont A, Malouinc F, Tousignant B, Jacksona PL (2015) Assessing motor imagery ability in younger and older adults by combining measures of vividness, controllability and timing of motor imagery. Brain Res 1597: 196-209.

30. Watanabe M, Takamatsu M (2014) Spatial perspective taking is robust in later life. Int J Aging Hum Dev 78: 277-297.

31. Rival C, Ceyte H, Olivier I (2005) Developmental changes of static standing balance in children. Neurosci Lett 376: 133-136.

32. Malouin F, Richards CL, Durand A (2010) Normal aging and motor imagery vividness: implications for mental practice training in rehabilitation. Arch Phys Med Rehabil 91: 1122-1127.

33. Mulder T, Hochstenbach JB, van Heuvelen MJ, den Otter AR (2007) Motor imagery: the relation between age and imagery capacity. Hum Mov Sci 26: 203-211.
34. Berg K (1989) Measuring balance in the elderly: preliminary development of an instrument. Physiother Canada 41: 304-311.

35. Watanabe M, Katagi R, Ishikawa K, Kawamura W (2008) Development of a Spatial Cognition Test for the Old Handicapped. Proceedings of the 20th Biennial Meeting of ISSBD, Vilnius, Lithuania.

36. Rosenberg I (1989) Summary comments: epidemiological and methodological problems in determining nutritional status of older persons. Am J Clin Nutr 50: 1231-1233.

37. Landi F, Liperoti R, Russo A, Giovannini S, Tosato M, et al. (2012) Sarcopenia as a risk factor for falls in elderly individuals: results from the ilSIRENTE study. Clin Nutr 31: 652-658.

38. Sodian B, Thoermer C, Metz U (2007) Now I see it but you don't: 14month-olds can represent another person's visual perspective. Developmental Sci 10: 199-204.

39. Mcdonald L, Stuart-Hamilton I (2002) Egocentrism in older adultsPiaget's three mountains task revisited. Educ Gerontol 28: 35-43.

40. Creem SH, Hirsch DT, Wraga M, Harrington GS, Proffitt DR, et al. (2001) An fMRI study of imagined self-rotation. Cogn Affect Behav Neurosci 1: 239-249.

41. Wraga M, Shephard JM, Churcha JA, Inatic S, Kosslyn SM (2005) Imagined rotations of self versus objects: An fMRI study. Neuropsychologia 43: 1351-1361.

42. Hasan KM, Walimuni IS, Abid H, Frye RE, Ewing-Cobbs L, et al. (2011) Multimodal quantitative magnetic resonance imaging of thalamic development and aging across the human lifespan: Implications to neurodegeneration in multiple sclerosis. J Neurosci 31: 16826-16832. 